\title{
Témoignages de Barrin de La Galissonière sur la situation interne de l'Amérique française
}

\section{Roland Lamontagne}

Volume 15, numéro 3, décembre 1961

URI : https://id.erudit.org/iderudit/302133ar

DOI : https://doi.org/10.7202/302133ar

Aller au sommaire du numéro

Éditeur(s)

Institut d'histoire de l'Amérique française

ISSN

0035-2357 (imprimé)

1492-1383 (numérique)

Découvrir la revue

Citer cet article

Lamontagne, R. (1961). Témoignages de Barrin de La Galissonière sur la situation interne de l'Amérique française. Revue d'histoire de l'Amérique française, 15(3), 333-343. https://doi.org/10.7202/302133ar d'utilisation que vous pouvez consulter en ligne. 


\section{TÉMOIGNAGES DE BARRIN DE LA GALISSONIËRE SUR LA SITUATION INTERNE DE L'AMÉRIQUE FRANÇAISE *}

A l'occasion de sa mission scientifique en Amérique du Nord, ${ }^{1}$ Joseph Bernard Chabert de Cogolin affirmait «qu'il y a bien des choses qu'on ne trouve pas dans les magazins d'une colonie presque naissante ». Il avait éprouvé maintes difficultés dont il faisait part à son supérieur, Barrin de La Galissonière. Les obstacles qu'il décrit, dans une lettre désabusée, retardaient les recherches qui devenaient, de ce fait, plus coûteuses. A Louisbourg, on n'avait pas reçu «d'ordre précis » de fournir à Chabert et à l'équipage « les choses absolument necessaires pour se garantir des rigueurs de l'hiver ». Comme il arrive souvent à un nouveau venu, Chabert ne peut franchir le réseau de protection mutuelle: «on manque de tout icy, même avec de l'argent à moins d'être lié de connoissance avec les particuliers de la Ville $\gg^{2}$ Dans ces conditions précaires de travail, Chabert craint d'être incapable d'accomplir sa mission scientifique et de déplaire au ministre. Il sollicite la protection de La Galissonière et la

* L'auteur tient à remercier le Conseil des Arts du Canada dont il a reçu, en 1961, une bourse de recherche qui lui a permis de compléter, aux Archives de France, sa documentation relative à Roland-Michel Barrin de La Galissonière. Il exprime sa gratitude à $M^{\text {me }}$ Marguerite Densky, bibliothécaire de l'université de Montréal, à $\mathrm{M}^{\mathrm{me}} \mathrm{G}$. Feuillebois, bibliothécaire de $\mathrm{l}^{\prime} O$ bservatoire de Paris et à $\mathrm{M}^{1 \mathrm{ll}} \mathrm{M}$. Chabrier, chef du service photographique de la Bibliothèque Nationale de Paris ainsi qu'à MM. Jean-M. Beauchemin, ptre, archiviste de l'Archevêché de Québec, Pierre Brunet, adjoint de l'archiviste du Canada, F. Dousset, adjoint du directeur général des Archives de France, Yves Laissus, archiviste-paléographe du Muséum national d'histoire naturelle de Paris, John $\mathrm{E}$. Pomfret, directeur de la bibliothèque Henry E. Huntington, San Marino, Californie, qui lui ont communiqué des renseignements et des microfilms de sources manuscrites.

1 Observations de Chabert dans l'Amérique du Nord, 1750 et 1751. Bibliothèque de l'Observatoire de Paris, B 5, 3, 9; G. Bigourdan, « Inventaire général et sommaire des manuscrits de la bibliothèque de l'Observatoire de Paris 》, Annales de l'Observatoire de Paris, 21 (Paris, 1895), 25.

2 Chabert à La Galissonière, Louisbourg, 12 décembre 1750, Archives de France, Marine III 1, I JJ, Registre $n^{\circ} 1$. 
permission de rentrer en France le plus tôt possible. Dans cet état psychologique, Bernard Chabert établissait peut-être une comparaison trop étroite entre la métropole et la colonie qu'il désirait quitter.

Contrairement à Chabert qui n'était que de passage en Nouvelle-France, Jean-François Gaultier s'y était établi à titre de médecin du roi. Il devint membre du Conseil supérieur et correspondant de l'Académie royale des Sciences. ${ }^{3}$ Tout en exprimant ses sentiments d'admiration à l'égard du gouverneur intérimaire, il insiste sur l'état de colonie presque naissante. Il est vrai qu'un aperçu de l'œuvre de colonisation intégrale de l'intendant Jean Talon permettrait d'infirmer une partie de ce témoignage de Gaulthier:

M. le marquis de La Galissonnière est le seul qui ait commencé à mettre les choses sur un bon pied. Le Canada a fait une très grande perte en le perdant. La vaste étendue de ses connoissances jointe à son grand amour pour le bien public et pour tout ce qui peut être utile à l'Etat l'auroit porté à établir solidement une colonie qui est presque naissante, et où on n'a presque rien fait depuis 150 ans. Ce qu'il y a de certain, c'est que ce qu'on y a fait est plustot dû au hasard et à une certaine routine qu'à la sagesse du gouvernement. ${ }^{4}$

En 1739, La Galissonière avait qualifié la colonie de «pays misérable $\gg{ }^{5}$ Hocquart ne s'exprime pas autrement en $1743 .{ }^{6}$ La guerre de la Succession d'Autriche provoque un accroissement de capitaux métropolitains destinés au Canada. Elle s'accompagne d'un relèvement du niveau de vie. Des observateurs tels que Pehr Kalm et Louis Franquet évoquent l'aisance de la société

3 Jean Torlais, éd., Réaumur (Paris, 1939), 17; Jacques Rousseau, «Michel Sarrazin, Jean-François Gaulthier et l'étude prélinnéenne de la flore canadienne》, Les Botanistes français en Amérique du Nord avant 1850 (Paris, 1957), 149-157; Observations botanico-météorologiques faites au Canada, par Gauthier [sic], de 1742 à 1748. Bibliothèque de l'Observatoire de Paris, A 7, 6, 643 .

${ }^{4}$ Gaulthier à Guettard, Québec, 21 octobre 1752, manuscrit 293, pièce 2, Muséum national d'histoire naturelle.

5 La Galissonière à Duhamel du Monceau, Rochefort, 3 avril 1739. Archives publiques du Canada, M.G. 18, G. 11. 1960), 66.

6 Jean Hamelin, Economie et société en Nouvelle-France (Québec, 
canadienne. Après une visite chez un habitant de Lachenaie, l'ingénieur Franquet pense que le roi devrait bien «charger un peu »des gens qui vivent aussi largement. ${ }^{7}$

A l'époque de l'administration intérimaire de La Galissonière, la colonie était peu peuplée. Faute de données exactes, il est possible de calculer une approximation à partir des recensements de 1739 et de $1754 .^{8}$ La population canadienne s'élevait à 42,701 âmes en 1739 et à 55,009 en 1754. En 1749, elle devait compter environ 50,000 habitants. La même année, la population française de la péninsule acadienne, de l'Ile Royale, de l'Acadie de terre ferme et de l'Ile Saint-Jean, au nombre de 16,000 h., se répartit comme suit: 9

Régions

Péninsule acadienne
Population $13,000 \mathrm{~h}$. $1,000 \mathrm{~h}$. $\begin{array}{ll}\text { Acadie de terre ferme [Nouveau Brunswick] } . \ldots \ldots \ldots . . . & 1,000 \mathrm{~h} . \\ \text { Isle Saint-Jean [Prince Edouard] } & 1,000 \mathrm{~h} .\end{array}$ $\begin{array}{ll}\text { Acadie de terre ferme [Nouveau Brunswick] } . \ldots \ldots \ldots . . . & 1,000 \mathrm{~h} . \\ \text { Isle Saint-Jean [Prince Edouard] } & 1,000 \mathrm{~h} .\end{array}$

Le ministre de la Marine juge bon d'accepter les ressortissants anglais, irlandais et écossais qui, après avoir été faits prisonniers, demandent à s'établir dans la colonie. ${ }^{10}$ C'est, penset-il, autant d'habitans acquis au Pays. »11

Selon une dépêche conjointe du gouverneur intérimaire et de l'intendant, douze ou quinze Irlandais catholiques ont sollicité la permission de s'établir au Canada; «quelques uns ont des Enfans dans les villages sauvages qui ont desja pris leurs mœurs, d'autres travaillent de quelques metier et sont gens de journée ».12

7 Guy Frégault, «Essai sur les finances canadiennes, 1700-1750 》, Revue d'histoire de l'Amérique française, 13 (septembre, 1959) : 179.

8 Recensements du Canada, 1665-1871 (Ottawa, 1876), 4: 60-61.

9 Ibid., 4: xxv.

10 « Le party de retenir en Canada les enfans anglois que nos sauvages $y$ menent ne pourroit estre avantageux qu'autant qu'on pourroit les $y$ fixer pour toujours afin d'augmenter par là le nombre des hab de la Colonie. 》 Maurepas à La Galissonière, Marly, 18 janvier 1748, AC, B, $87: 54$.

11 \& Il ne paroit pas dangereux de garder dans la Colonie les Irlandois et les Ecossois catholiques qui y seront conduits, et qui demanderont a y rester. 》Id. à Id. et à Hocquart, Marly, 23 janvier 1748, AC, B, 87: 86 . 12 La Galissonière et Hocquart à Maurepas, Québec, 24 octobre 1747 , Archives publiques du Canada, microfilm des Archives des Colonies, série C11A, 87: 150 . 
La Galissonière et Bigot appliquent à regret les instructions du ministre concernant l'immigration irlandaise; leur réponse est tendancieuse. "Nous avons suivi vos intentions a l'egard des Irlandois catholiques qui ont voulu rester en ce pays, mais tous catholiques qu'ils sont, la pluspart sont trés mauvais sujets et dont la Colonie ne tirera jamais grand profit. ${ }^{13}$ De tels préjugés sont à l'origine de vies sacrifiées et de souffrances accumulées. Ils s'écartent de la vérité qui réside dans l'accord entre le jugement et les faits par opposition aux opinions préconçues que l'imagination, l'amour-propre, les passions et les fétiches d'ordre ethnique, social ou intellectuel contribuent à former. On en trouve aussi des exemples dans les ouvrages historiques. Sous forme de généralisation, cette affirmation de Roland Mousnier découle d'un préjugé racial, de l'ignorance des faits ou de l'interprétation erronée de la condition humaine: «Les Français traitaient les Indiens avec douceur, cherchaient à les comprendre, à les élever, à les assimiler. Les Espagnols faisaient des lois pour les protéger. Les Anglo-Saxons, ici comme partout, étaient racistes d'instinct, sinon de théorie. Ils avaient une répulsion pour les Indigènes et tendaient à les détruire. ${ }^{14} \mathrm{Com}-$ me on peut le constater, il arrive à un analyste d'une réalité sociale de manquer de sens critique et d'effectuer une extrapolation abusive à partir d'un nombre minime de faits soumis à une interprétation partiale.

Grâce à l'adoption d'esclaves sauvages, les Indiens cherchaient à accroître leur population. La Galissonière et Hocquart voulurent mettre fin à cette pratique. Ils avaient proposé à la métropole de racheter, avec le consentement des propriétaires indigènes, ces esclaves qu'ils déporteraient aux Antilles. Sous prétexte de l'effet néfaste que produirait sur les Indiens l'expatriation érigée en système, Maurepas refusait cet expédient, en $1748 .{ }^{15}$

13 Id. et Bigot à Id., Québec, 29 septembre 1748, AC, C11A, 91: 33. 14 Le XVIIIe siècle. Révolution intellectuelle, technique et politique, 1715-1815. Histoire générale des civilisations publiée sous la direction de Maurice Crouzet ( 7 vol., Paris, 1953-1957), 5: 315.

15 Maurepas à La Galissonière, 18 janvier 1748, $\mathrm{AC}, \mathrm{B}, 87$ : 54-55. 
La Galissonière a jugé sévèrement les Canadiens, «trop paresseux et trop glorieux $\gg .^{16}$ Cette expression est contenue dans une lettre signée conjointement avec l'intendant Bigot. Le qualificatif de paresseux apparaît avec une certaine fréquence dans la correspondance du gouverneur intérimaire qui ne prisait pas le manque d'intérêt scientifique des Canadiens. "Il ne m'est venu aucune graine de Canada cette année. Ce sont tous des paresseux. » ${ }^{17}$ La Galissonière confiait à Gradis que les Canadiens «sont si indolens et si paresseux qu'ils laissent tout perdre et ce n'est qu'en continuant d'envoier [ «les semences de différents fruits »] qu'on peut se flatter qu'il leur restera quelque chose $\gg .^{18}$

La traite des fourrures exigeait de la part des colons un travail moins systématique que celui de l'agriculture. Les habitants des Illinois ont été «bien paresseux et se sont plus donnés a la traitte qu'au bled $» 19$ écrit La Galissonière à Vaudreuil. Selon un mémoire du roi adressé à La Jonquière, plusieurs Canadiens sont satisfaits du profit du commerce des fourrures et délaissent l'agriculture. ${ }^{20}$ De plus, la ville exerce une attraction sur la population rurale. En avril 1749, François Bigot publie une ordonnance qui empêche les paysans de s'installer à Québec. ${ }^{21}$

La Galissonière veut s'assurer une voie de communication entre Québec et le Cap-Breton en interdisant aux Britanniques l'accès du territoire situé à l'ouest de la Nouvelle-Écosse. «Si nous abandonnions a l'Angleterre ce terrain qui comprend plus de cent quatre-vingt lieues de côtes, c'est-à-dire presqu'autant qu'il y en a de Bayonne a Dunkerque, il faut renoncer à toute 91: 26.

${ }^{16} \mathrm{La}$ Galissonière et Bigot, Québec, 26 septembre 1748, AC, C11A, G. 11 .

17 Id. à Duhamel du Monceau, Brest, 3 février 1745, APC. M.G. 18,

18 Paris, 16 janvier 1754, Rapport de l'archiviste de la province de Québec (1944-1945), 279.

19 Paris, 23 janvier 1751, Huntington Library and Art Gallery, Loudoun Papers, 269.

20 Mémoire du Roy pour servir d'instructions au $\mathrm{S}^{\mathrm{r}} \mathrm{M}^{\mathrm{is}}$ de La Jonquiere chef d'escadre des armées navales Gouverneur lieutenant général de la Nouvelle-France. Versailles, 30 avril 1749. Harvard College Library.

21 Guy Frégault, La Société canadienne sous le régime français. Publication de la Société historique du Canada (Ottawa, 1954), 13. 
communication par terre de Canada avec l'Acadie et l'isle Royale et à tout moïen de secourir l'une et de reprendre l'autre. ${ }^{22}$ En septembre 1748, le gouverneur intérimaire stimulait l'émigration des Acadiens du territoire britannique. A cette fin, il avait expédié à l'lle Saint-Jean l'émissaire Benoist ${ }^{23}$ et Charles Deschamps de Boishébert à la rivière Saint-Jean. ${ }^{24} \mathrm{~A}$ l'automne de 1749, La Jonquière se disposait à envoyer le chevalier Louis Luc de La Corne à l'isthme de Chignectou. ${ }^{25}$

Maurepas rappelait à l'intendant le drame de la migration acadienne:

Vous êtes instruit depuis longtemps des vues qu'on a d'engager des Acadiens a transporter leurs establissements a l'isle Royale et principalement a l'isle $\mathrm{S}^{\mathrm{t}}$ Jean. Les arrangements que le gouvernement d'Angleterre projette pour envoyer des Anglois s'establir en Acadie pourront donner des facilités pour l'exécution de ces vues. Outre les desgouts qu'on cherchera peut estre à donner aux anciens françois, il pourra y en avoir qui voudront eviter le mélange des deux nations. Non seulement il faudra bien accueillir ceux qui se presenteront pour s'establir soit à l'isle Royale, soit à l'isle $\mathrm{S}^{\mathrm{t}}$ Jean ou même en Canada, mais encore il sera à propos d'en attirer autant qu'il sera possible a l'isle $\mathbf{S}^{\mathrm{t}}$ Jean dont vous savez que l'etablissement seroit si interessant pour l'isle Royale. ${ }^{26}$

Guy Frégault a dégagé les divers aspects de la stratégie française dans les années postérieures au traité d'Aix-la-Chapelle et, notamment, il a mis en relief le rôle des missionnaires sur le plan politique. ${ }^{27} \mathrm{Au}$ sujet de l'abbé Le Loutre, La Galissonière avouait à Mgr Henri-Marie du Breil de Pontbriand, en 1753:

22 La Galissonière à Rouillé, Québec, 25 juillet 1749, AC, C11A, 93 : 125.

23 Rapport concernant les archives canadiennes pour l'année 1905 (Ottawa, 1909) , 2:344 (Appendice N.).

${ }^{24} \mathrm{La}$ Galissonière à Rouillé, Québec, 25 juillet $1749, \mathrm{AC}, \mathrm{C} 11 \mathrm{~A}, 93: 123$.

25 La Jonquière à Id., Québec, 9 octobre 1749, AC, C11A, 93: 181. Library.

26 Maurepas à Bigot, Versailles, 11 avril 1749, Harvard College

${ }_{27}$ Guy Frégault, « La déportation des Acadiens », Revue d'histoire de l'Amérique française, 8 (décembre, 1954) : 309-358. 
J'espere que le voiage qu'a fait icy [Paris] M. Le Loutre fera un bon effet pour les pauvres acadiens, pour qui je m'emploie de mon mieux, mais avec moins d'effet que je ne desirerois. Je regarde cet excellent missionaire comme la meilleure protection que puisse avoir cette malheureuse partie de notre nation, a qui cependant elle fait honneur et a qui elle peut être très utile. ${ }^{28}$

Le ton de cette autre lettre permet d'apprécier l'intérêt de La Galissonière à l'égard du problème acadien:

La situation des habitans de l'Acadie me fait une peine extrême, et j'ai fait ce que j'ai pu pour qu'on prit des mesures pour l'adoucir mais je ne sai point quelle reussite ont eu mes sollicitations, ni même quelle reussite auroit ce que j'ai proposé en cas qu'on l'exécutat. Une grande partie du meilleur des Acadiens vient de leur attachement a leurs anciennes terres qui est fort naturel et de l'indolence et même de l'espece de découragement avec lequel ils se portent a travailler a de nouveaux établissemens ce qui est encore plus naturel. Leurs missionnaires seuls peuvent affoiblir ces obstacles. ${ }^{29}$

L'Acadie revêt, aux yeux de La Galissonière, une importance particulière et Saint-Domingue, qui ne fait pas partie de la Nouvelle-France, devrait se situer au centre des préoccupations métropolitaines. ${ }^{30}$ La Galissonière tient à rappeler au début de son mémoire sur les Antilles françaises qu' * il y a aussi peu de françois instruits de la valeur réelle de $S^{t}$ Domingue qu'il y en a eu peu qui n'ayent exagéré celle de Louisbourg $\gg^{31}$ Il dénonce les abus. Selon lui, le commun dénominateur de l'administration française en Amérique, c'est l'exploitation au profit personnel des autorités coloniales: «L'Employ [des fonds métropolitains] en a été aussi infidèle que dans les autres colonies. $\gg 32$

28 La Galissonière à Pontbriand, Paris, 18 mai 1753, Archives de l'Archevêché de Québec, G III-112.

29 Id. à Id., Paris, 19 mai 1752, Ibid., G III-106.

30 Mémoire contre les Projets des Anglois, sur nos Colonies Méridionales de l'Amérique, par M. de la Galissonière. APC. Bibliothèque Nationale, Fonds français, 11340, folio 3.

31 Ibid., 4 .

32 Ibid., 6. 
La Galissonière propose de transformer les esclaves nègres en militaires et d'en prélever les frais sur la caisse des suppliciés qui constitue un fonds de remboursement destiné aux propriétaires des esclaves criminels que la justice condamne à mort. «Cet impot, explique-t-il, se leve comme la capitation en taxant chaque nègre. la taxe est de quarante sols il $\mathrm{y}$ a environ 150000 nègres dans la Colonie, ainsy elle monte par chaque année à $300000 £$ Et souvent il n'en sort pas un sols, pour l'objet qui a donné lieu a cette imposition. L'Employ de l'argent est secret, c'est l'intendant qui le tire des mains du Receveur; et l'on ignore a qui il en rend compte. ${ }^{33}$

Les ports de Saint-Domingue où abordent chaque année trois cent cinquante navires marchands chargés des denrées du royaume et qui rapportent en retour celles du crû de l'île «forment au moins au Total un article de 150 millions ${ }^{34} \mathrm{~A}$ l'exception des marchands forains ou des négociants des places maritimes, on a une faible idée de la richesse de Saint-Domingue qui constitue, selon La Galissonière, «la plus féconde branche, ou pour mieux dire, le véritable tronc ${ }^{35} \mathrm{du}$ commerce métropolitain. En juin 1751, La Galissonière cherche à encourager les planteurs de coton des Antilles françaises; il conseille de leur faire parvenir la liste des prix du coton dans toutes les villes du royaume et principalement dans celles où se trouvent les grandes manufactures qui en font usage. Il précise de «faire mention des droits qui se payent en france sur cette matière première afin qu'on ne puisse pas faire accroire aux cultivateurs de Coton que ces droits sont énormes comme on l'a fait fort souvent $\gg .{ }^{36}$

La correspondance privée de La Galissonière contient des détails relatifs à l'aspect économique de la colonisation. En 1741, La Galissonière recommande d'avoir recours aux entrepreneurs. «Je suis bien aise, écrit-il à Duhamel du Monceau, qu'on ait trouvé de beaux bois au Missisipi mais c'est bien loin

33 Ibid., 11.

34 Ibid., 4.

35 Ibid., 5.

36 Bibliothèque Nationale, Mazarine 2840, ff. 198-199. 
pour l'apporter dans des Flûtes et la Colonie est et sera longtems trop faible pour entreprendre d'y construire, outre qu'il ne pourroit sortir du Fleuve que d'assez petits vaisseaux. Tout ce qu'on pourroit faire ce seroit d'engager les marchands par quelque gratification et l'esperance de la durée des vaisseaux à y faire bâtir, cela fortifieroit le pays et le Roi par la suitte pourroit profiter pour luy mesme des ouvriers qu'ils auroient dressés. ${ }^{37}$

$\mathrm{Au}$ cours de son administration intérimaire de la NouvelleFrance, La Galissonière a été limité par le mercantilisme. Dans le cadre de ce système économique, les colonies produisent les matières brutes, exportées sur la métropole, qui reviennent manufacturées; elles constituent des annexes économiques de la France. Maurepas le rappelle clairement à La Galissonière qui avait contribué à établir des manufactures d'étoffes de laine: "il ne convient de les tolérer qu'autant qu'elles ne pourront pas nuire au débouchement de celles de France, et c'est aussi par cette raison qu'il ne faut pas les laisser multiplier $\gg{ }^{\mathbf{3 8}}$

En 1748, La Galissonière et Bigot dans une dépêche conjointe affirment que le coût de la vie est élevé. ${ }^{39}$ L'année suivante, ils réclameront cent cinquante charpentiers. Les produits d'importation française coûtent cher. La colonie a même manqué de marchandises de traite et de sel. Le naturaliste et chimiste Duhamel du Monceau devait communiquer à La Galissonière un procédé de fabrication de sel que l'on utilisait dans quelques provinces du royaume démunies de salines. ${ }^{40}$ Le ministre de la Marine tirait profit de l'habileté scientifique et technique du gouverneur intérimaire: «je suis bien persuadé que M. le $\mathrm{C}^{\text {te }}$ de La Galissonière fera usage de son zele et de ses connoissances pour contribuer a consolider l'establissement des constructions dans la Colonie ».41 La construction navale avait

$37 \mathrm{La}$ Galissonière à Duhamel du Monceau, à bord du Tigre en rade de Toulon, 7 octobre 1741. Muséum national d'histoire naturelle.

38 Maurepas à La Galissonière, 6 mars 1748, AC, B, 87: 212. $91: 24$.

39 La Galissonière et Bigot, Québec, 26 septembre 1748, AC, C11A,

40 Maurepas à La Galissonière, 6 mars 1748, AC, B, 87: 212.

41 Id. à Id. et Hocquart, Marly, 23 janvier 1748, AC, B, 87: 82. 
ralenti. ${ }^{42}$ En 1748 , le roi ordonnait de mettre en chantier «un second $V^{\text {au }}$ de la mesme force que le $\mathrm{S}^{\mathrm{t}}$ Laurent ${ } .^{43}$

Après avoir pris connaissance d'un mémoire relatif au commerce du blé, que lui avait communiqué $\mathrm{Mgr}$ de Pontbriand, La Galissonière exprime ses vues sur le marché de cette céréale:

Je pense qu'on peut s'en tenir a la connoissance du prix du Bled pour savoir s'il faut donner ou refuser des permissions d'en sortir. On fait, dit-on, depuis longtemps l'équivalent de cela en Angleterre quand le bled est a bas prix, on donne tant par minot a ceux qui le font sortir, et quand il monte trop, on donne tant par minot a ceux qui en font entrer. Cette retribution seroit peut être en Canada sujette a abus, mais elle ne me paroit pas necessaire. le plus difficil est peut être de fixer ce prix moien du Bled. mais je ne vois pas qu'il y ait de grands inconveniens a se tromper en prenant tousjours l'opinion la plus favorable pour faire rester beaucoup de Bled dans le pays. ${ }^{44}$

La Galissonière avoue humblement les limites de ses connaissances en économie: «en voila plus que je n'en devrois dire sur une matiere que je ne sai gueres ». L'administration des finances qui ressortissait à l'intendant ${ }^{45}$ échappe au coup d'œil que nous avons jeté sur l'état de la colonie d'après la correspondance de La Galissonière. La Cour insiste sur la nécessité de réduire les débours et de n'en faire que d' "indispensables ${ }^{46}$ Elle néglige la défense militaire. En 1749, les vingt-huit compagnies du Canada groupaient environ 700 troupiers. "Il n'y a pas une seule compagnie de complète et . . . il faudroit plus de la moitié de soldats qu'il y en a pour qu'elles le fussent sur le pied de 50 hommes. ${ }^{47}$

${ }^{42}$ Jean Hamelin, Economie et société en Nouvelle-France (Québec, $1960), 117$. 87: 81.

43 Maurepas à La Galissonière et Hocquart, 23 janvier 1748, AC, B,

44 La Galissonière à Pontbriand, Paris, 18 mai 1753, Archives de l'Archevêché de Québec, G III-112.

$45 \mathrm{Au}$ sujet de l'administration financière de la colonie, consulter G. Frégault, 《Essai sur les finances canadiennes, 1700-1750 》, Revue d'histoire de l'A mérique française, 13 (septembre, 1959) : 157-182.

46 Maurepas à Hocquart, 6 mars 1748, AC, B, 87: 192.

47 La Jonquière à Rouillé, Québec, 20 septembre 1749, AC, C11A, $93: 88$. 
Vu la pénurie de techniciens au Canada et l'incertitude relative à la qualité du fer, le gouverneur intérimaire n'avait pas cherché à hâter, en 1748 , l'établissement d'une "fonderie d'artillerie de fer» qui, à son avis, stimulerait le commerce entre la Nouvelle-France et les Antilles. «La Martinique et $\mathbf{S}^{t}$ Domingue, pensait-il, paieroient en partie les canons qui s'y porteroient d'ici [Canada] en melasses et en guildives pour le magazin du Roy qui en consomme assés considerablement. » ${ }^{48}$

Ces témoignages sur la situation intérieure de l'Amérique française avaient pour but de mettre en relief le vif intérêt que Barrin de La Galissonière portait à l'essor des Antilles françaises et de la Nouvelle-France.

Université de Montréal.

\section{ROLAND LAMONTAGNE}

48 La Galissonière à Maurepas, Québec, 18 octobre 1748. Microfilm F, AC, C11A, 91: 154 .

\section{OUVRAGES REÇUS :}

G. JACQUEMYNS, Langrand-Dumonceau, promoteur d'une puissance financière catholique, I-II. Université libre de Bruxelles,, 1960.

E. B. OSLER, The man who had to hang Louis Riel. Longmans Green \& Co., Toronto, 1961.

Ulane BonNEL, La France, les Etats-Unis et la guerre de course (1797-1815). Nouvelles éditions latines, Paris, 1961.

VICTOR MARIE DU PONT, Journey to France and Spain 1801. Cornell University Press, Ithaca, New York, 1961.

K. G. Davies, M.A., Editor, Peter Skene Ogden's Snake Country Journal 1826-2\%. London, The Hudson's Bay Record Society, 1961. 